\title{
In situ analysis of lignins in transgenic tobacco reveals a differential impact of individual transformations on the spatial patterns of lignin deposition at the cellular and subcellular levels
}

\author{
Matthieu Chabannes ${ }^{1, \dagger}$, Katia Ruel ${ }^{2,1}$, Arata Yoshinaga ${ }^{3, \dagger}$, Brigitte Chabbert ${ }^{4}$, Alain Jauneau ${ }^{5}$, Jean-Paul Joseleau ${ }^{2}$ and \\ Alain-Michel Boudet ${ }^{1, *}$ \\ ${ }^{1}$ UMR CNRS/UPS 5546, Signaux et Messages Cellulaires chez les Végétaux, Pôle de Biotechnologie Végétale, \\ 24 Chemin de Borde-Rouge - BP 17 Auzeville - 31326 Castanet-Tolosan, France, \\ ${ }^{2}$ Centre de Recherches sur les Macromolécules Végétales, CNRS, BP 53, 38041 Grenoble Cedex 09, France, \\ ${ }^{3}$ Laboratory of Structure of Plant Cells, Division of Forest and Biomaterials Science, Kyoto University, \\ Kyoto 606-8502 Japan, \\ ${ }^{4}$ Laboratoire de Biochimie, INRA centre de Reims, 2 Esplanade R. Garros, BP 224, 51686 Reims cedex 2, France, and \\ ${ }^{5}$ Institut Fédératif de Recherche Fr40 'Signalisation Cellulaire et Biotechnologie végétale', Pôle de Biotechnologie \\ végétale, BP 27, 31326 Castanet Tolosan cedex, France
}

Received 29 May 2001; revised 26 July 2001; accepted 3 August 2001.

*For correspondence: (fax +33 (0)5 621935 02; e-mail amboudet@smcv.ups-tlse.fr).

${ }^{\dagger}$ These authors contributed equally to this work.

\begin{abstract}
Summary
Using tobacco transgenic lines altered in the monolignol biosynthetic pathway and which differ in their lignin profiles we have evaluated lignin deposition at the cellular and subcellular levels using several microanalytical techniques. Surprisingly, whereas a Cinnamoyl CoA reductase (CCR) down-regulated line with a strong decrease in lignin content exhibited an overall reduction in lignin deposition in the walls of the different xylem cell types, this reduction was selectively targeted to the fibers in a double transformant (down-regulated for both CCR and Cinnamyl alcohol dehydrogenase (CAD)) displaying a similar degree of global lignin content decrease. Fiber and vessel secondary walls of the transgenic tobacco line homozygous for the ccr antisense gene (CCR.H) down-regulated plants were dramatically destructured, particularly in the S2 sublayer, whereas the deposition of lignins in the S1 sublayer was not significantly modified. In contrast, cell wall organization was slightly altered in xylem cells of the double transformant. The relative distribution of non-condensed and condensed units in lignin, evaluated microscopically with specific antibodies, was differentially affected in the transgenics studied and, in a general way, a drop in non-condensed lignin units ( $\beta-0-4$ interunit linkages) was associated with a loss of cohesion and extensive disorganization of the secondary wall. These results demonstrate that lignification is tightly and independently regulated in individual cell types and cell wall sublayers. They also show that down-regulation of specific genes may induce targeted changes in lignin structure and in spatial deposition patterns of the polymer.
\end{abstract}

Keywords: lignification, lignin deposition, transgenic tobacco, wall layers, vessels and fibers.

Introduction

Quantitative and qualitative analyses of lignins in transgenic plants modified for the activity of enzymes involved in the phenylpropanoid and monolignol pathways have mainly considered lignified tissues as a whole, without specifically evaluating the changes induced in different types of cells. This is clearly due to the difficulty of separating or probing in situ the different cell types of xylem, a heterogeneous tissue.

Secondary xylem comprises several types of lignified cells particularly in woody dicotyledons: water conducting 
tracheary elements (vessels members and tracheids) and mechanically supporting elements (fiber-tracheids, libriform fibers and sclereids) (Lev-Yadun, 2000). In herbaceous plants, such as tobacco, vessels and fibers are the main lignified components of xylem. It is generally assumed that the composition of lignins differs according to the cell type; generally, vessels are enriched in $G$ units and fibers in S units (Fergus and Goring, 1970). This has been demonstrated, for example, in Arabidopsis stems in which vascular bundles rich in vessels containing preferentially $\mathrm{G}$ units in their lignin, alternate with interfascicular fibers particularly enriched in S units (Chapple et al.; 1992)

At a more subtle level, the deposition of lignin has been investigated by direct examination of lignin in the cell wall through non-destructive methods. Terashima et al. (1986, 1993) have shown, by microautoradiography of xylem sections after labeling with appropriate precursors, that lignification occurs in three distinct stages preceded by deposition of carbohydrates in the different layers of the secondary wall $\left(S_{1}, S_{2}, S_{3}\right)$. Moreover, the three kinds of monolignol units seem to be incorporated at different stages of the cell wall formation in the following order: hydroxyphenyl $(H)$, guaiacyl $(G)$ and syringyl (S) units (Terashima and Fukushima, 1989). These observations of a sequential deposition of lignin monomeric units are in accordance with those found by UV microscopic spectrophotometry (Musha and Goring, 1975).

All these different data strongly suggest that lignification is tightly regulated during the developmental programs leading to different cell types and that lignin deposition within the wall is a highly organized process. Lignin composition of each individual cell appears to be controlled by a complex array of gene expression in the differentiating cells or in adjacent cells as suggested, for example, by the recent results of Chen et al. (2000). These authors have shown that, in transgenic Populus tremula $x$ $P$. alba hybrids, the expression of ccoaomt promoter-Gus fusions - is observed in differentiating xylem preferentially in contact rays adjacent to the vessels but is not detectable in other ray cells or in fibers. These data strongly suggest different controls of monolignol biosynthesis in the different xylem cell types.

It is clear that the understanding of the regulatory mechanisms controlling the nature and the spatial deposition of lignins within different cell types and within the different layers of the wall is still very fragmentary. However, this information is a prerequisite to manipulating, in a cell orientated way, the lignins of higher plants. Knowledge of lignin subcellular distribution is also important because it may give an insight into the supramolecular relationships that occur in the cell wall between cellulose, hemicelluloses and lignins.

Apart from histochemical examination of stem sections stained with classical staining reagents, no in situ characterization of the lignification patterns of cell walls in transformed plants has been carried out. In this work, we report that different modifications of the lignin synthesis pathway through genetic engineering results in specific patterns of lignin deposition at the cellular and the subcellular levels which have been evaluated by UV microspectrophotometry (Fukazawa, 1992) and immunocytochemical techniques (Joseleau and Ruel, 1997).

\section{Results}

In previous work (Halpin et al., 1994; Piquemal et al., 1998; Chabannes etal., 2001), we have demonstrated that different transgenic tobacco lines transformed with independent antisense constructs corresponding to the $c c r$ and cad gene or containing the two antisense genes, exhibit different lignin profiles. Interestingly, plants down-regulated for Cinnamoyl CoA reductase (CCR) activity or for both CCR and Cinnamyl alcohol dehydrogenase (CAD) activities strongly differed in their morphology and the structural integrity of their vessels despite a strong reduction in lignin content (up to $50 \%$ ) (Chabannes et al., 2001).

\section{Characteristics of vessel elements in different transgenic lines}

These results were confirmed and extended by image processing and analysis of vessels from tobacco stems at the microscopic level using the natural fluorescence of lignins under UV light (Table 1). The following parameters were evaluated in the vessels of control and different transgenic lines: thickness of the walls, area, roundness and deformation (estimated by the ratio $\mathrm{M}: \mathrm{m}$ ) of the cells. These two last parameters are related to the shape of the vessels.

Table 1. Measurement of different geometrical parameters of xylem vessels. The area, the roundness (R) (see Experimental procedures), the ratio $\mathrm{M}: \mathrm{m}$ (ratio of major axis to minor axis of the vessels on transverse sections) and the thickness of the cell wall were determined on the vessels from four different tobacco lines (Wt: wild type; CCR.H: transgenic line homozygous for the ccr antisense gene; CAD.H: transgenic line homozygous for the cad antisense gene; Dt: double transformant resulting from the sexual crossing of the two previous lines and hemizygous for the two transgenes). Data (mean values \pm S.E.) were obtained on 50 different cells per line and analysed using Student's $t$-test

\begin{tabular}{lclll}
\hline & area $\left(\mu \mathrm{m}^{2}\right)$ & $\mathrm{R}$ & $\mathrm{M}: \mathrm{m}$ & thickness \\
\hline $\mathrm{Wt}$ & $1476 \pm 65$ & $1.22 \pm 0.01$ & $1.26 \pm 0.02$ & $2.6 \pm 0.08$ \\
CCR.H & $510 \pm 18^{*}$ & $1.56 \pm 0.02^{*}$ & $1.7 \pm 0.04^{*}$ & $2.7 \pm 0.12$ \\
CAD.H & $1134 \pm 63^{*}$ & $1.23 \pm 0.01$ & $1.26 \pm 0.03$ & $2.6 \pm 0.08$ \\
Dt & $1029 \pm 99^{*}$ & $1.27 \pm 0.01^{*}$ & $1.4 \pm 0.02^{*}$ & $2.9 \pm 0.10$ \\
\hline
\end{tabular}

${ }^{*}$ means statistically different 
In contrast to Arabidopsis irregular xylem mutants (irx), which exhibit a decrease in cellulose content and thinner cell walls (Turner and Somerville, 1997), no significant differences were observed concerning the thickness (Table 1) of the vessel walls of the different transgenic lines. However, the genetic transformations had a dramatic impact on the area and the shape of the vessels. The average cell area, for example, was three times lower for the transgenic tobacco line homozygous for the $c c r$ antisense gene (CCR.H) line than in the control (Table 1). This decrease could also be observed but to a lesser extent for the transgenic tobacco line homozygous for the cad antisense gene (CAD.H) line and for the double transformant down-regulated for both CCR and CAD (Dt). As already shown (Piquemal et al., 1998) xylem vessels of the homozygous CCR line (CCR.H) are collapsed, losing much of their roundness, and are dramatically deformed, whereas those of Dt were only slightly affected. In order to understand the reason for these differences, we have investigated in a comparative way, the deposition of lignins in the different cell types using several techniques.

\section{Histochemical surveys}

Histochemical staining with phloroglucinol- $\mathrm{HCl}$. Figure 1 shows an histochemical survey of the different transgenic lines using the Wiesner reaction (phloroglucinol $-\mathrm{HCl}$ ) on microtome sections of tobacco stems, in order to obtain a better resolution than with hand-made sections. In comparison with the control line (Figure 1a), the CAD.H depressed line (Figure 1c) exhibited a slight increase in Wiesner staining in vessel and fiber secondary walls probably due to an increase in the proportion of cinnamaldehydes incorporated into the lignins (Yahiaoui etal., 1998). In contrast, in the CCR.H depressed line (Figure 1b) all the different cell types of the xylem were only very weakly stained. Dt showed a red purple color comparable with Wt only in vessel secondary walls and compound middle lamella but very faint staining in the fibers (Figure 1d). Since Wiesner staining is not supposed to be a strict quantitative technique and since differences could be potentially due to accessibility rather than changes in end group concentrations, these observations were then confirmed by UV microspectrophotometry.

\section{Ultraviolet microscopic spectrophotometry}

Figure 2 shows ultraviolet absorption spectra in vessel secondary walls (V-SW), fiber secondary walls (F-SW) and cell corner middle lamella (FF-CC) in wild type and transgenic lines. Spectra were averaged on the cells whose lignification (indicated by increase in absorbance values in Figure 3) was already completed.
In comparison with the wild type, the CCR.H depressed line exhibited an important decrease in absorbance values and a variation in absorption maxima whatever the cell type examined. In the CAD.H depressed line, cell walls displayed similar absorption maximum to the wild type in V-SW (277.5 nm), F-SW (275 nm), FF-CC (275-277.5 nm). No changes in absorbance value or in absorption maximum were observed in V-SW and FF-CC in Dt compared with corresponding cells of the wild type. In contrast, the absorbance value decreased for F-SW and the absorption maximum was shifted to a shorter wavelength $(270 \mathrm{~nm})$ compared with wild type $(275 \mathrm{~nm})$. This indicated that F-SW of the Dt might be richer in syringyl units than F-SW in wild type since it is known that the enrichment in $S$ units cause a shift in absorption maximum to shorter wavelengths (Musha and Goring, 1975).

Besides the absorption around $280 \mathrm{~nm}$ corresponding to non-conjugated units in lignins, a shoulder around 325$330 \mathrm{~nm}$ was more pronounced in all transgenic lines. This phenomenon could reflect accumulation of wall bound phenolics whose absorption maximum is in the range of 320-330 nm.

In Figure 3, the UV absorbance maximum of lignins is plotted against the distance from cambial zone. The absorbance values were significantly decreased in V-SW, F-SW and FF-CC in CCR.H depressed line. No significant variation was found in the CAD.H depressed line. In contrast, in the case of Dt, the decrease in absorbance was only found in F-SW and no significant change occurred in V-SW and FF-CC.

This cytological comparison of lignin content between wild type and transgenic lines was consistent with the results of chemical analysis. Reduction in lignin content was chemically demonstrated in both CCR.H and the Dt but not in the CAD.H line. Ultraviolet microspectrophotometry enabled us to clarify the difference between CCR.H and the Dt. The reduction in lignin content occurred in all cell wall types in CCR.H line and only in F-SW in Dt. This is also consistent with the histochemical observations (Figure 1).

Further studies were then performed at the ultrastructural level on control and the two transgenic lines with a decreased lignin content (CCR.H and Dt).

\section{Ultrastructural morphology and lignin topochemistry}

Cell wall ultrastructural morphology. General contrasting of polysaccharides by the periodate oxidation-thiocarbohydrazide-silver proteinate method (PATAg) (Ruel etal., 1981) allows the visualization in transmission electron microscopy (TEM) of all the cell walls, whether they are lignified or not.

Figure 4(a) represents a cross section through a part of fiber and vessel walls of the wild-type plant. The classical 


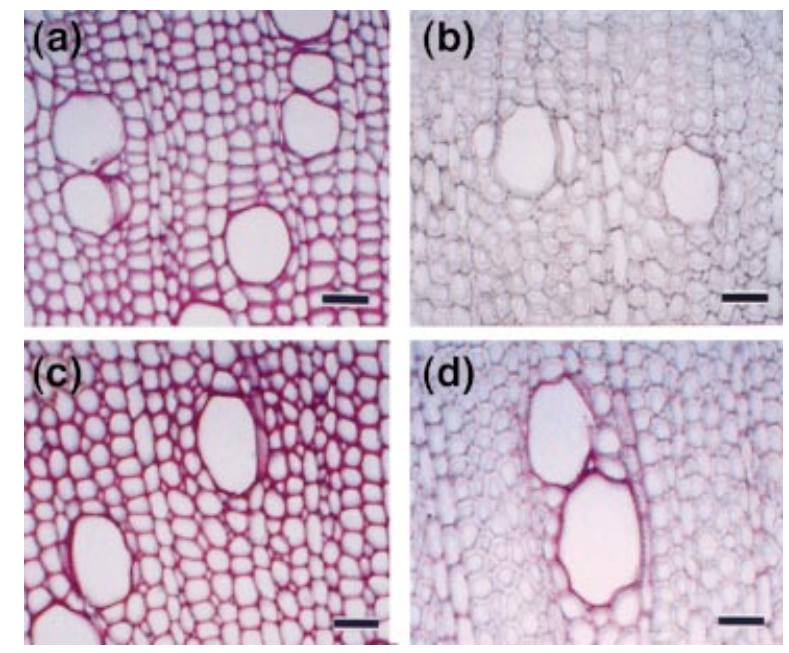

Figure 1. Light micrographs of stem cross sections of the different tobacco lines after Phloroglucinol- $\mathrm{HCl}$ staining.

Microtome-made transverse stem base sections are shown after phloroglucinol staining for the four following tobacco lines, Wt: control (a); CCR.H (b), CAD.H (c) and Dt: CAD.H $\times$ CCR.H plants (d).

Bar represents $50 \mu \mathrm{m}$.

subdivision of the secondary walls in sublayers S1, S2 and S3 in fibers and in three sublayers $(1,2,3)$ in the vessel wall is recognizable in these cells and underscored by an enhanced reactivity to PATAg of the borders between sublayers. The junction areas between adjacent cells (the so-called compound middle lamella), and cell corners are highly reactive to periodate oxidation, as shown by their black staining.

The CCR.H transformant was clearly identified by an extensive disorganization of sublayers S2 (in fibers) and 2 (in vessels) (Figure 4b) compared with the Wt (Figure 4a). This alteration is primarily characterized by a general loosening of the entire secondary wall thickening corresponding to S2 in fibers and sublayers 2 and 3 in vessels. The inset in Figure 4(b1) shows that the observed loosening is due to a disorganization of the cellulose framework where cellulose microfibrils appeared individualized. In contrast, the sublayers S1 (in fibers) and 1 (in vessels) appeared unaltered, showing the same cohesion as that observed in the normal plant.

In the Dt, the organization of fiber and vessel walls was similar to that of $\mathrm{Wt}$. The different sublayers could be easily differentiated and a more detailed observation showed an enhancement of the reactivity of S3 to the PATAg staining (Figure 4c).

Patterns of lignification in the cell walls of transgenic tobacco plants. We then carried out immunolabeling in TEM using antibodies directed, respectively, against condensed and non-condensed guaiacyl-syringyl (GS) lignin
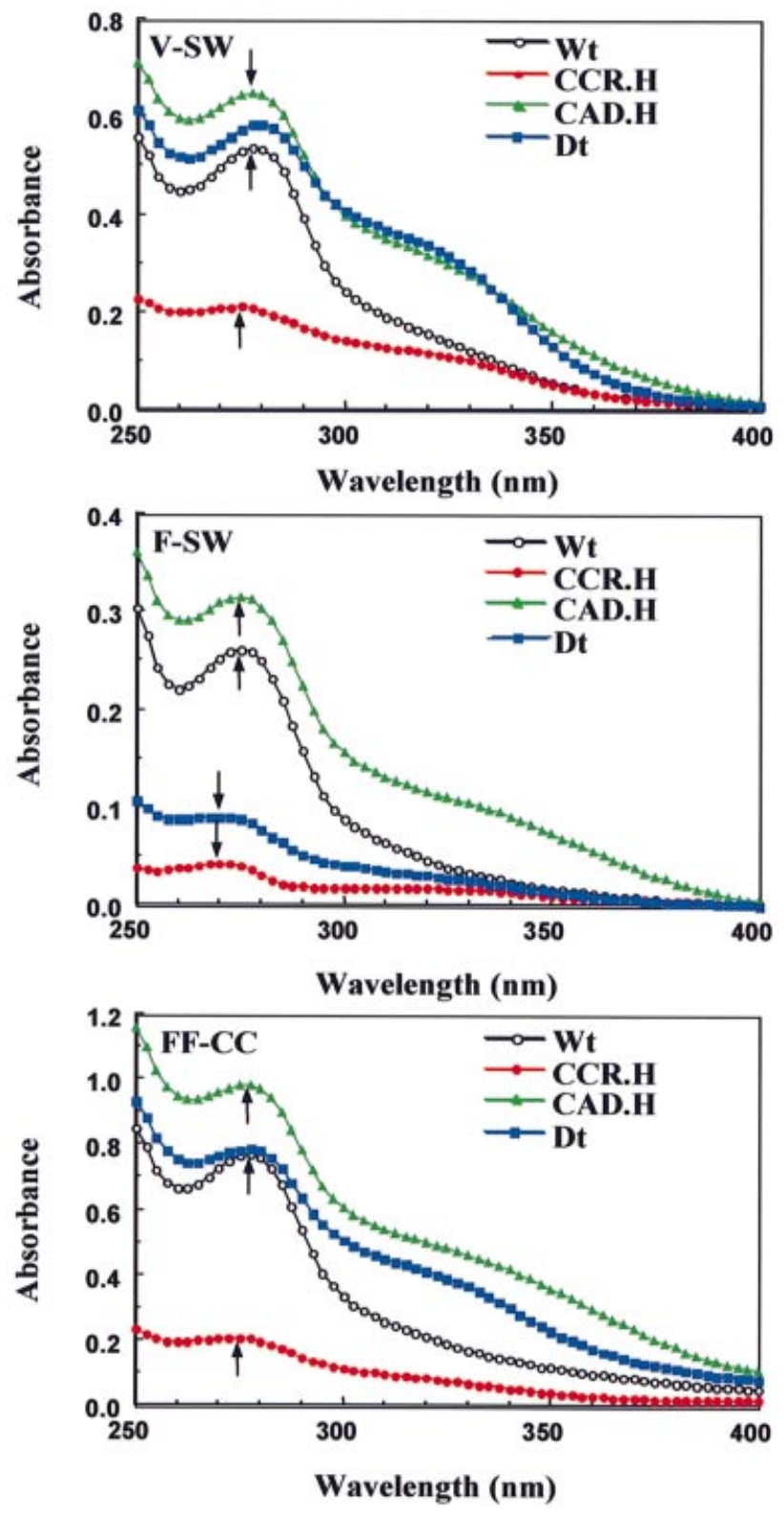

Figure 2. Ultraviolet absorption spectra of vessel secondary walls (V-SW), fiber secondary walls (F-SW) and cell corner middle lamella located between fibers (FF-CC) in wild type (Wt), CCR.H depressed line, CAD.H depressed line and Dt (CAD.H $\times$ CCR.H).

Arrows indicate absorption maxima of the spectra. The slight increase in absorbance observed for different samples when compared with wild type (for example in V-SW of CAD.H and Dt) are not significant and are due to differences in section thickness.

subunits. Considering the above observation that fibers and vessels were differently affected by the transformation, these tissues were examined separately. Figure 5 gives the results obtained with pre-immune sera showing no staining on the cell walls as already observed in previous studies on maize internodes (Joseleau and Ruel, 1997). 

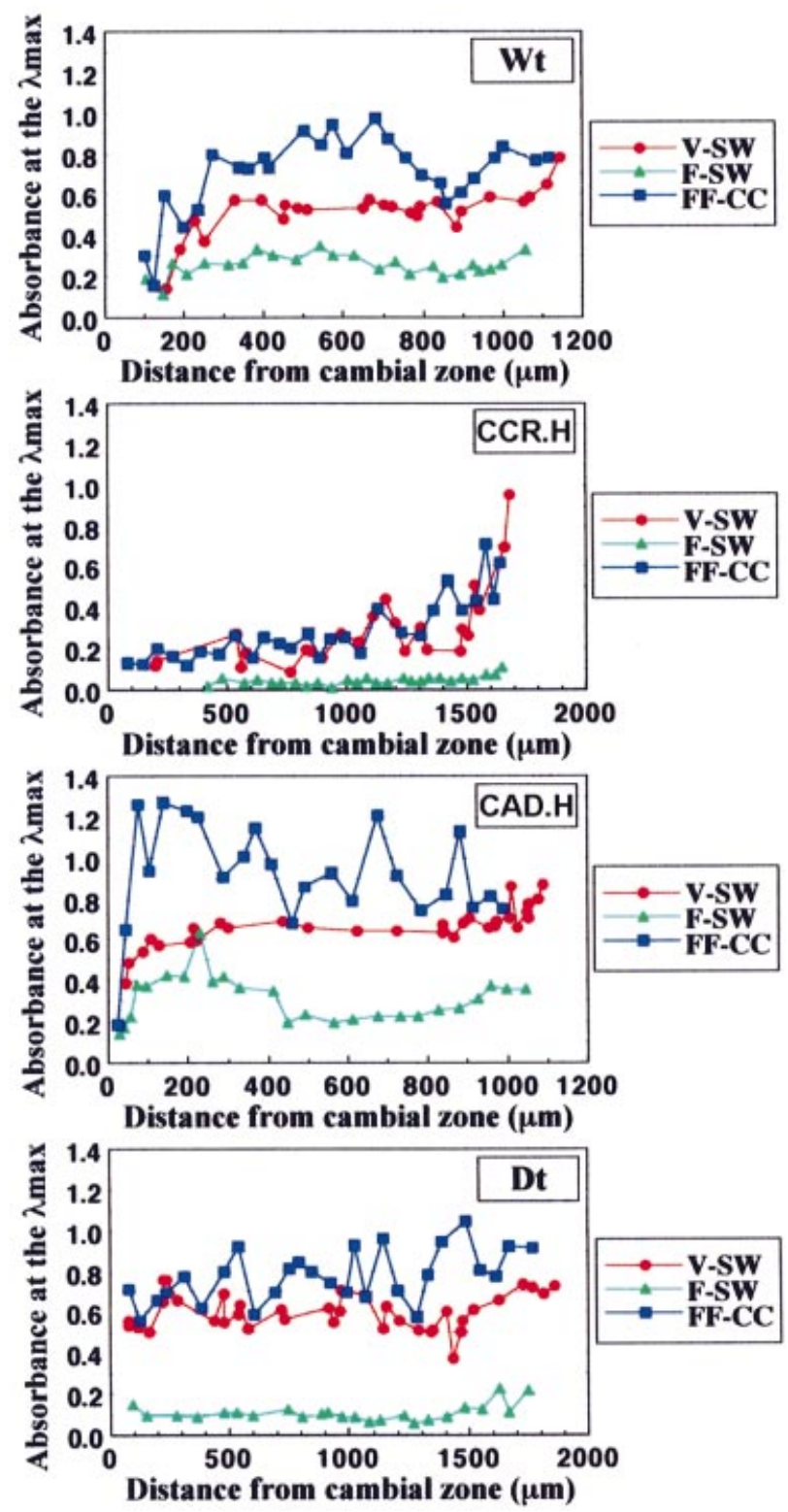

Figure 3. UV absorbance of secondary walls of different cell types (vessels (V-SW), fibers (F-SW) and cell corner middle lamella located between fibers (FF-CC)) at different distances from the cambial zone in wild type $(\mathrm{Wt}), \mathrm{CCR} . \mathrm{H}$ depressed line, CAD.H depressed line and $\mathrm{Dt}$ (CAD. $\mathrm{H} \times \mathrm{CCR} . \mathrm{H})$.

Distances from cambial zone may vary according samples depending on xylem width.

Topochemical distribution of lignins in fibers of Wt, CCR.H and Dt. All TEM images of wild type plants labeled for non-condensed GS lignin subunits indicated that these types of lignin structures were well represented in the fiber cell walls of the Wt. However, the distribution varied between the three sublayers as illustrated in Figure 6(a). Non-condensed GS subunits were abundant and homogeneously distributed in the wall layers. It should be noted that these epitopes were not significantly found in middle lamellae and cell corners showing that these anatomical zones did not harbor the same type of lignin as the secondary walls of fibers.

In the case of the CCR.H, distinctive modifications in the patterning of lignification were observed (Figure 6b). The non-condensed GS epitopes had become concentrated in the S1 layer and the outer part of S2 of the transformant, with only a few gold particles visible in the de-structured inner S2 layer.

In the Dt plants (Figure 6c), the distribution of the noncondensed GS epitopes in S1 and S2 did not markedly differ from the normal plant. These results show that in the $\mathrm{Dt}$, the fibers incorporated non-condensed lignin in almost the same way as in the wild type plant.

Immunolabeling of condensed GS subunits in the $\mathrm{Wt}$ was mostly detected in the S1 sublayer of the fiber walls (Figure 6d) and was essentially absent from S2 and S3. In the CCR.H down-regulated plant, condensed epitopes were also principally distributed in the $\mathrm{S} 1$ sublayer of the secondary wall but they spread on the outer part of S2 (Figure 6e). The loosely organized inner S2 sublayer was unlabeled. In Dt, fiber walls were totally unlabeled (Figure 6f). This observation clearly indicated that an important reduction in the synthesis of condensed lignin subunits happened in the secondary walls of Dt fibers.

Topochemical distribution of lignins in vessel of Wt, CCR.H and $\mathrm{Dt}$. In vessel walls of $\mathrm{Wt}$, the non-condensed subunits, were in general mostly localized in sublayer 2 (Figure 7a). In the CCR.H down-regulated plant, these lignin subunits were found concentrated in sublayer 1 (Figure 7b) as already shown for fibers (Figure 6b). They were almost absent from the two other sublayers 2 and 3, which displayed altered ultrastructural morphology. In Dt, noncondensed GS lignin subunits were abundantly represented and the intensity of the labeling varied depending on the vessel type (Figure 7c): in protoxylem, non-condensed GS epitopes were homogeneously distributed, whereas in metaxylem, these epitopes were mainly restricted to the sublayers 1 and 3 .

The condensed lignin subunits were abundant in the vessel walls of the Wt. Their distribution was different in the three sublayers and they were more abundant in sublayers 1 and 3 (Figure 7d). In the CCR.H depressed line, the condensed GS subunits were more localized to sublayer 1 and to the outer part of sublayer 2 (Figure 7e). In Dt, labeling was very weak in all sublayers, but appeared to be above background in sublayer 1 (Figure 7f).

Comparing the CCR.H down-regulated and the Dt plants, which both showed a similar decrease in their total lignin content, it appears that the transformations induced dramatic and specific changes in the different lignin types and in their topochemical distribution. 

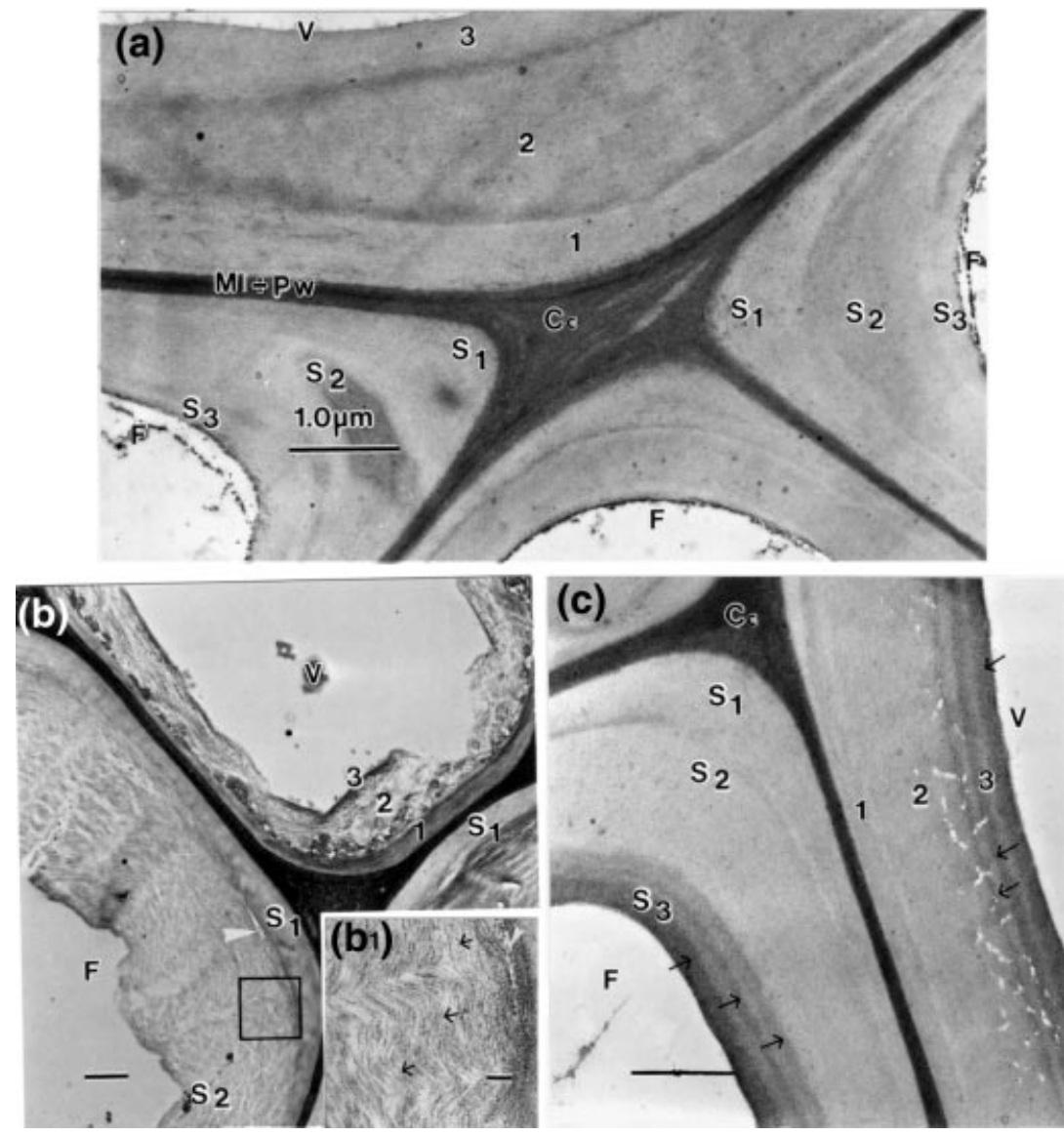

Figure 4. Ultrastructural organization of the xylem cell walls in the wild type, the CCR.H down-regulated transformant and the Dt: CAD. $\mathrm{H} \times \mathrm{CCR} . \mathrm{H}$ plants. PATAg staining on ultra-thin transverse sections.

(a) Wild-type. Fiber and vessel walls exhibit a general staining covering the different layers and sublayers. In fiber walls, S1 (outer layer), S2 (middle layer) and S3 (innermost layer), are identifiable. In vessel wall, three concentric sublayers are visible (noted 1, 2, 3 from outer layer to inner layer). Cell corner and middle lamellae are strongly reactive to the PATAg staining.

(b, inset b1) CCR.H depressed line. A dramatic loosening in the cell wall architecture of S2 can be seen both in fibers and vessels - in fibers, a concentric sublayering appeared, ending at a clear separation of cellulose microfibrils in S2. Arrow-heads indicate weak points between S1 and S2. The inset (b1) shows an enlarged view of S2, underlying the unmasking of cellulose microfibrils which appear individualized (small arrows).

(c) Dt (CAD.H $\times$ CCR.H). A general good cohesion of the different sublayers appears, similar to that observed in Wt. Compared with the $\mathrm{Wt}$, the thickness of the fiber cell walls has increased and the inner part of S2 and $\mathrm{S} 3$ has become more reactive to the PATAg staining (arrows). Cc, cell corner; F, fiber; $\mathrm{Ml}+\mathrm{Pw}$, compound middle lamella; S1, S2 and S3, outer-, middle and innermost layers of the secondary wall of fibers; $\mathrm{V}$, vessel; 1, 2, 3, outer, middle and innermost layers of the secondary wall of vessels. Bars in $A, B$ and $C=1.0 \mu \mathrm{m}$; in the inset, $B 1=0.2 \mu \mathrm{m}$.
Non-condensed GS lignin subunits are present in both fibers and vessel walls of the $\mathrm{Wt}$ and of the $\mathrm{Dt}$. These substructures are almost entirely absent from the disorganized parts of the secondary walls of the CCR.H downregulated line both in fibers and vessels.

Condensed GS lignin subunits, or at least specific types of condensed units, are present in both Wt and CCR.H depressed lines. They can be considered practically absent from fiber walls and absent or only very slightly represented in vessel walls in the Dt.

\section{Discussion}

In previous lignin genetic engineering experiments, structural modification of lignin has been analyzed by chemical and instrumental analysis. However, whether genetic transformation affects various types of cells and the different layers of the wall in the same way has not been clarified since the results of chemical analysis represent a global evaluation of mixed cell types. Thus, in this study, to clarify the spatial effect of the transformations, histochemical analyses, ultraviolet microspectrophotometry and immunocytochemistry were applied to evaluate in situ lignin distribution and structure in different types of cells and in different layers of the walls.

\section{Different transformation events may differentially affect spatial deposition of lignins in xylem cell types}

Histochemistry and in situ UV microspectrophotometry have shown that in the CCR.H depressed line, a dramatic decrease in lignin content in all cell wall types resulted in collapsed vessels. In contrast, Dt plants with a decrease in lignin content only in F-SW exhibited limited alterations of the xylem organization (Chabannes et al., 2001).

The explanation for the differential effects observed in the two types of transformants is not clear at the moment. We can suggest that different targeted gene modifications could influence secondarily, through changes in the concentration of phenolic intermediates, the expression pattern of genes more specifically involved in the deposition of lignins in the different cell types. Recent results from Chen et al. (2000) have clearly shown Caffeoyl coenzyme A 3-O methyltransferase (CCoAOMT) gene expression in a single specific cell type: the contact ray cells that are 

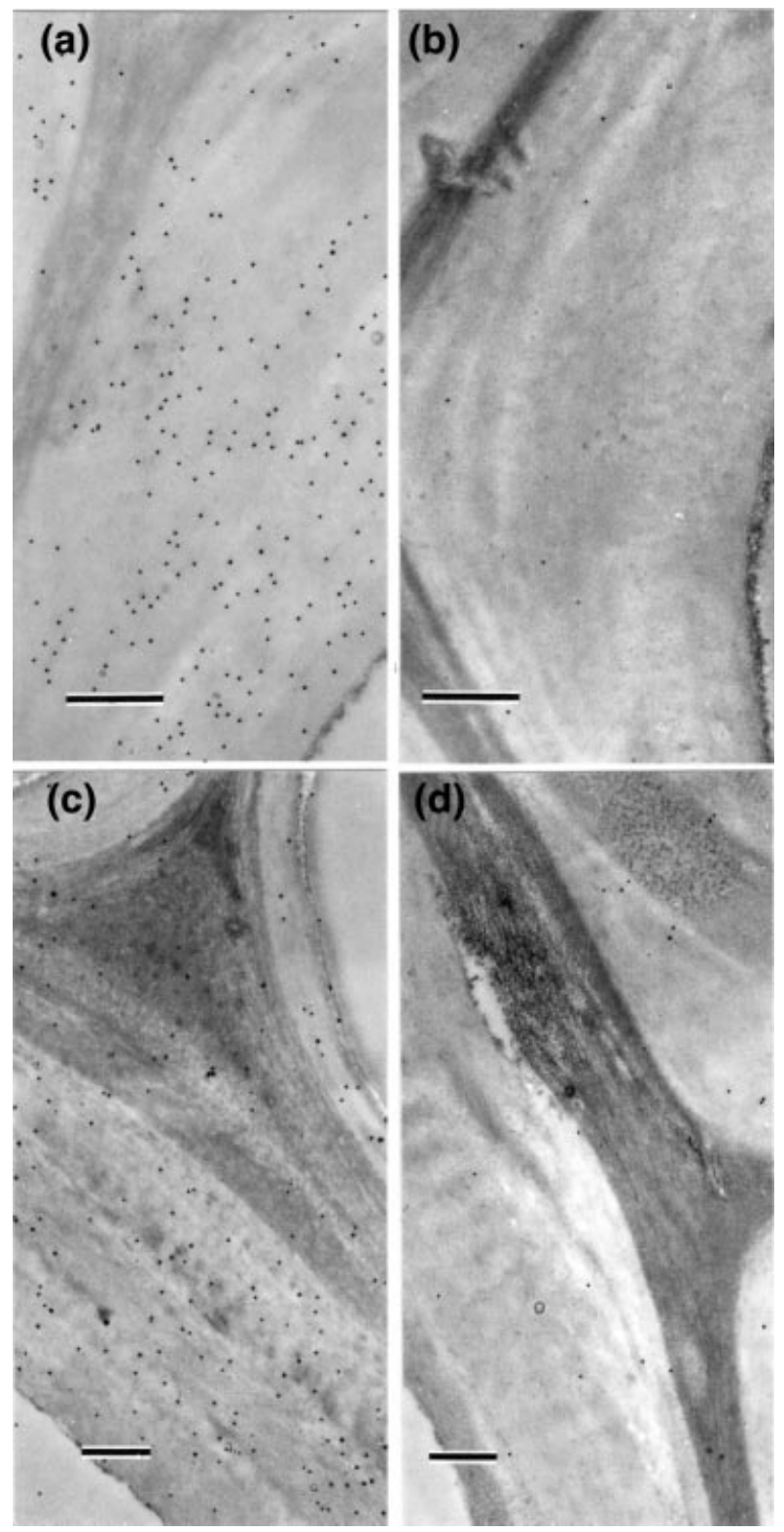

Figure 5. Controls. The gold particles $(5 \mathrm{~nm})$ were silver enhanced. As a result, the size of silver grains may vary from one preparation to another due to temperature and time. However, the actual number of grains remains unchanged.

(a-b) Control for the antiserum directed against-non-condensed GS lignin subunits in fiber cells. (a) Wt incubated with the antimixed-noncondensed GS subunits antiserum: the walls are clearly labeled. (b) $\mathrm{Wt}$ incubated with the corresponding preimmune serum: only a few gold particles-non-specifically deposited are visible.

(c-d) Control for the antiserum directed against condensed GS lignin subunits in vessel and fiber cells (vessel wall is localized at the bottom left of both photographs, other walls correspond to fibers). (c) $\mathrm{Wt}$ incubated with the antimixed condensed GS subunits antiserum: numerous gold particles. (d) Wt incubated with the corresponding preimmune serum: only a few gold particles-non-specifically deposited are visible. Bars in $a-d=0.5 \mu \mathrm{m}$.

connected to vessels. This strictly localized expression could be related to the relative enrichment of vessels in $\mathrm{G}$ units.
Whatever the mechanisms involved, the limited qualitative modifications of lignins in Dt underlined by NMR analyses (Chabannes etal., 2001) and the maintained deposition of non-condensed enriched lignins in vessels of the Dt are likely responsible, at least in part, for the structural and functional integrity of these conducting elements and of the normal phenotype of the plants. From a biotechnological point of view and despite the functional importance of lignification in fibers, recently evidenced by genetic approaches (Zhong and Taylor, 1997), these results suggest that lignins could be specifically decreased in fibers, at least moderately, without adverse effects on development.

\section{Modified expression of genes involved in the synthesis of monolignols resulted in significant changes in structure and topochemical distribution of lignins}

The main features, which can be deduced from Figures 6 and 7, revealed dramatic differences between the CCR.H down-regulated line and the Dt. Indeed, the lignification patterns are quite distinct both at the qualitative and spatial levels. Lignins enriched in non-condensed units are dramatically decreased in fibers and vessels after downregulation of CCR alone but do not display significant changes in the Dt in comparison with the control. In contrast, lignins enriched in condensed units, which are almost absent in the Dt plants (Figures $6 f$ and $7 f$ ), were present in the CCR.H line at similar (Figures $6 \mathrm{e}$ and 7e) or higher levels than in the control. At the spatial level, noncondensed lignins, which are widely distributed within the different sublayers of fibers and vessels in the wild type and the Dt, were restricted to the S1 sublayer in the CCR.H down-regulated line. Thus, in addition to a differential effect of the specific transformation events on the deposition of lignins in different cell types of the xylem, a specific impact on the structure of lignins and on their distribution in the walls of vessels and fibers can be observed.

Thus, for a similar global reduction in lignin content, the decrease concerns more specifically the non-condensed units (CCR.H line) or the condensed units of lignins (Dt). These results are in good agreement with previous chemical analyses of these engineered lignins showing a decrease in thioacidolysis yield (drop in non-condensed units) in the CCR.H line but a stability of this yield in the Dt (Chabannes et al., 2001). In CCR.H plants, the reduction of lignin deposition mainly affected the S2 and S3 layers of fibers and layers 2 and 3 of vessels and was accompanied by severe anatomical alteration of these regions of the cell walls. However, as indicated by our results, other wall sublayers $(\mathrm{S} 1,1)$ and other types of lignins (condensed GS substructure) are less affected by the transformation. The rationale of the distinctive chemical characteristics of 

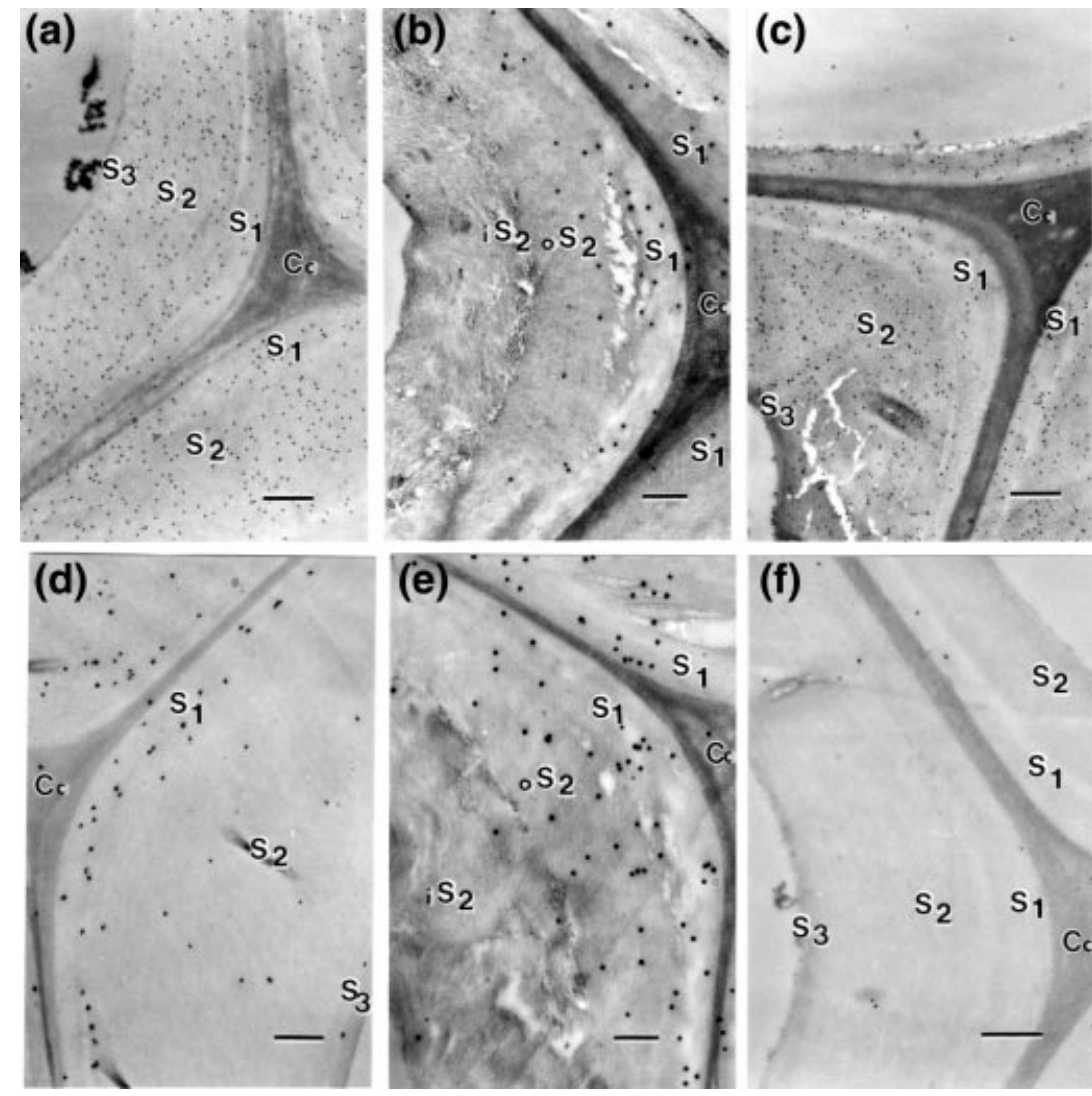

Figure 6. Immunocytochemical localization of non-condensed and condensed mixed guaiacyl-syringyl lignin subunits in fibers from Wt, CCR.H depressed and Dt (CAD.H $\times$ CCR.H) lines. As in Figure 5, the gold particles $(5 \mathrm{~nm})$ were silver enhanced. (a-c) Labeling for non-condensed GS lignin subunits. (a) In Wt, gold particles cover the three sublayers of the secondary wall of fibers, they are absent from middle lamella and cell corner. (b) In CCR.H depressed line: gold particles have become mostly localized in S1 and partly in outer part of S2 (oS2). non-condensed epitopes are absent in the loosened inner part of S2 (iS2). (c) In the Dt, an abundant and homogeneous deposit of gold particles is seen on the entire S2. S1 is less labeled, particularly in the part adjacent to the cell corner. As in Wt, gold particles are absent from middle lamella and cell corner. (d-f) Labeling for condensed GS lignin subunits. (d) In Wt, gold deposits are restricted to the S1 sublayer. (e) In the CCR.H depressed line: gold labeling has become concentrated in $\mathrm{S} 1$ and in the outer part of S2 (oS2). A few gold particles are deposited in the cell corner. (f) In the Dt, no gold deposits can be detected, either in S1 or in $\mathrm{S} 2$, or in middle lamella or cell corner. Cc, cell corner; F, fiber; MI, middle lamella; S1, S2 and S3, outer-, middle and innermost layers of the secondary wall; iS2, oS2, inner and outer parts of S2 sublayer; V, vessel; 1 , 2,3 , outer, middle and innermost layers of the secondary wall of vessels. Bars in a-f $=0.5 \mu \mathrm{m}$.
CCR.H and Dt lignins is not clear at the moment. However it is known that the type of lignins synthesized in cell corners and secondary walls are strongly influenced by the respective geometry of the randomly arranged carbohydrate polymers present in the former and the oriented arrangement of microfibrils in the second. A direct consequence of the random environment is to give rise to a bulk polymerization of lignin monomers between which the condensed linkages are favoured. On the other hand, the narrow microfibrillar secondary wall environment induces an endwise type of polymerization that leads to the predominance of non-condensed linkages (Roussel and Lim, 1995).

One of the characteristics of this work was the exploitation of different techniques of lignin analysis based on specific and independent properties of the polymer (reactivity of cinnamaldehyde residues for phloroglucinol- $\mathrm{HCl}$ staining, UV absorbance of aromatic rings of lignins for UV microspectrophotometry, immunological reactivity of structural units of lignins). These techniques provide complementary information which has rarely been collected in the same plant material. However, the comparison of individual results has identified potential discrepancies. For example, a normal deposition of lignins in vessels of $\mathrm{Dt}$ is evidenced by phloroglucinol- $\mathrm{HCl}$ and UV absorbance but immunocytochemistry shows a drop in condensed unit enriched lignins which are almost absent in these conducting elements. This apparent contradiction could be explained by the poorly defined specificity of the selected immunological probe obtained against the model polymer simulating the bulk (condensed units) polymerization mode. These antibodies could reveal a specific type of interunit linkage or alternatively other chemical groups associated with lignin condensed units (e.g. free phenolic groups: Jacquet et al. (1997)).

Although it is not yet completely clear what epitopes are being recognized by the antibodies, they allow us to see dramatic differences in the distribution of specific lignin substructures in the different transgenic lines and this additional approach confirms the specificity of qualitative changes induced by different transformation events.

\section{Significant changes also concerned cell walls micromorphology and ultrastructural organisation}

Detailed anatomical investigation by TEM of the tobacco transformants revealed that combined antisense downregulation of CCR and CAD activities or of CCR activity alone resulted in slight or marked alterations of xylem organisation. The most dramatic cell wall alteration is by 
Figure 7. Immunocytochemical localization of non-condensed and condensed mixed guaiacyl-syringyl lignin subunits in vessels from $\mathrm{Wt}$, CCR.H repressed and $\mathrm{Dt}$ (CAD.H $\times$ CCR.H) lines. As in Figures 5 and 6 , the gold particles $(5 \mathrm{~nm})$ were silver enhanced.

(a-c) Labeling for non-condensed GS lignin subunits. (a) In the $\mathrm{Wt}$, the wall of this vessel from metaxylem exhibits heterogeneous labeling on the three sublayers with the most important labeling within the middle sublayer 2 where the vessel wall is adjacent to the neighbour cell. As in fibers, gold deposits are absent from middle lamella and cell corner. (b) In the CCR.H down-regulated transformant, the labeling is weak and concentrated on the sublayer 1. (c) In the Dt, an abundant and homogeneous deposit of gold particles can be seen on the wall of protoxylem, whereas gold deposits are the most abundant in layers 1 and 3 in the wall of metaxylem.

(d-f) Labeling for condensed GS lignin subunits. (d) In the $\mathrm{Wt}$, the distribution of the gold particles varies between sublayers 1-3. It is particularly abundant in 1 and 3 . (e) In the CCR.H down-regulated line, the labeling is present in the sublayer 1 and in the outer part of the sublayer 2. (f) In the Dt, no significant gold deposits can be seen on any of the three sublayers.

Cc, cell corner; MI, middle lamella; 1, 2, 3, outer, middle and innermost sublayers of the secondary wall of vessels; PX, vessel of the protoxylem; VM, vessel of the metaxylem.
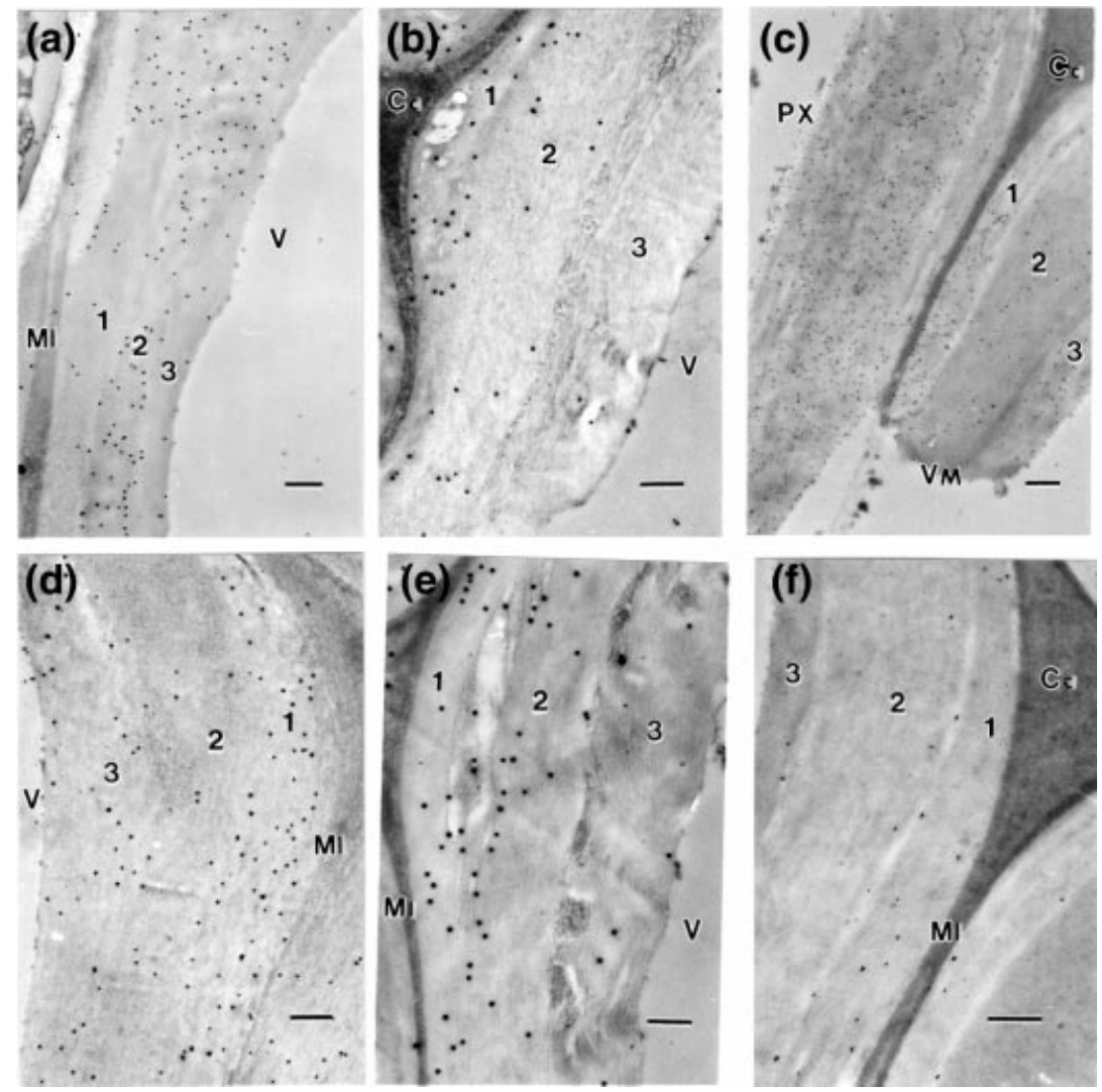

far that resulting from CCR inhibition which specifically affected the S2 sublayer of fibers and sublayer 2 of vessels but not the $\mathrm{S} 1$ and 1 sublayers. This unaltered aspect of S1 and 1 in fibers and vessels, besides the severely disorganised S2 and 2 secondary wall thickenings, suggests that there is a distinct control of lignification during secondary wall sublayers formation.

The depletion of lignification in the S2 and 2 sublayers associated with the alteration of the wall ultrastructure, constitutes a strong indication that lignin plays an active role in the secondary wall assembly. Indeed, the results of Figure 4(b1) clearly show that the cellulose microfibrils are individualized in the absence of the lignin polymer which normally should assume an adhesive role between the polysaccharide components of the wall. These results show the importance of lignin in the cohesion of the cellulose-hemicellulose matrix. They also show that the reduction in lignin content is not the only factor responsible for the cohesion since the CCR.H line and Dt, which both have comparable reductions in lignin content, displayed different repercussions in their secondary wall formation and cohesion. This suggests that cohesion between cellulose-hemicellulose microfibrillar elements of the secondary wall might involve a specific molecular type of lignin. This view was verified by immunological labeling and pointed out the important role of noncondensed unit enriched lignins in the conservation of structural integrity of vessels and fibers. The different behaviour of individual transformants could mean that beyond the synthesis of monolignol, their polymerization at the site of lignin deposition in the extracellular matrix is also precisely organized. In this latter step of cell wall assembly the physico-chemical factors such as the matrix effect due to polysaccharides environment (Siegel, 1957), and their polyelectrolyte structure (Houtman and Atalla, 1995) have also to be considered, during lignin polymerization and deposition, in addition to monolignol production.

It is also interesting to draw a parallel between the specific occurrence of lignins in the $\mathrm{S} 1$ sublayer of the secondary wall of CCR.H down-regulated tobacco plants and the specific occurrence in the same S1 sublayer of Forsythia intermedia cell walls of dirigent (monomers binding) sites (Gang etal., 1999). In the CCR.H downregulated plants, a shortage in lignin precursors is accompanied by a preferential location of lignins in the vicinity of the putative initiation sites (progenitorial lignin) where the organization of coupling could be facilitated. These 
observations are also in agreement with the location of the initial stages of lignin deposition as already described by Donaldson (1994).

Thus, two types of consequence seem to result from the genetic modulation of enzymes controlling lignin monomers biosynthesis. The first, which is evidenced through chemical analysis, concerns lignin content and monomeric composition, and the second, which affects the quantity and the quality of lignin synthesized in a particular site, may alter cell wall assembly and ultrastructural organization.

All together it is clear that the drop in non-condensed units of lignins and their restricted localization in the sublayer 1 are responsible for the collapse of vessels in the CCR.H down-regulated line. In contrast, the uniform distribution of an apparently stable equipment in these non-condensed units in the different sublayers of Dt vessels may allow them to maintain their structural integrity.

In conclusion, our results show that spatial deposition of lignins can be strongly altered by induced changes in the activity of key enzymes in monolignol synthesis. The data reported here provide evidence that different types of cells within the xylem have independent and precise regulatory mechanisms of lignin synthesis. This is particularly illustrated by the distinctive characteristics of fibers and vessels in the plant down-regulated for both CCR and CAD activities. These results suggest that induced, targeted modification of lignin synthesis in specific cell types should be possible in the future for biotechnological applications.

Various changes were also observed at the level of secondary wall sublayers and specific immunological probes revealed subtle differences in the qualitative patterning of lignification in the transformed plants. These observations, correlated to the alteration of xylem cells, demonstrate that lignins are important for the structural integrity of lignified secondary walls and that non-condensed structures play a major role in the cohesion of secondary wall layers. It becomes clear that not only biosynthesis but also polymerization of monolignols, or at least the supply of monolignols to different polymerization zones, are likely regulated to bring about the co-ordination essential for correct assembly of lignified secondary walls.

\section{Experimental procedures}

\section{Material}

The wild type and three transgenic tobacco lines were examined: CAD.H, a CAD down-regulated line homozygous for the cad antisense transgene, CCR.H, a CCR down-regulated line homozygous for the $c c r$ antisense transgene and CAD.H $\times$ CCR.H, a double transformant $(\mathrm{Dt})$ down-regulated for both $\mathrm{CCR}$ and $\mathrm{CAD}$ activities. The different analyses were performed on the basis of the stems of mature plants (just before flowering) grown in culture room. All the details concerning the obtention and the culture of these lines have been described in the accompanying paper (Chabannes etal., 2001).

\section{Methods}

Fluorescence microscopy and cell imaging. Hand made stem sections were examined with an inverted microscope (DM IRBE, Leica) and images acquired using a CCD camera (Colour Coolview, Photonic Science, UK) were processed and analyzed (Image Pro-Plus, Media Cybernetics, MD, USA). The area, the roundness and the $\mathrm{M}$ : $\mathrm{m}$ ratio were used as geometrical parameters to estimate changes in the xylem vessel shape. The roundness of the cells was determined by the formula: (perimeter) $)^{2} / 4$.pi.area (circular cells will have a roundness equal to 1 , whereas other shapes will have a roundness higher than 1). The M: $m$ ratio corresponds to the ratio between the length of the major axis and the minor axis of a given cell; the larger the ratio, the larger the deformation of the xylem vessels. In addition, the thickness of the wall vessels was measured to estimate quantitative changes in cell wall deposition. Data (mean values $\pm \mathrm{SE}$ ) were analyzed using Student's $t$-test. A $P$-value $<0.01$ was considered statistically significant.

Histochemical staining. Stem cross sections of $20 \mu \mathrm{m}$ thick were cut from wild type and the transgenic lines with a freezing microtome (Microm) equipped with a steel knife. These sections were subjected to the Wiesner reaction (phloroglucinol-HCl) (Adler etal., 1948). Sections were observed under a light microscope just after staining.

Ultraviolet microscopic spectrophotometry. Small blocks were dehydrated through an ethanol series and embedded in methyl-and-butyl (1:1) methacrylate resin. From the embedded specimens, $1 \mu \mathrm{m}$ thick cross sections were cut with a diamond knife mounted on an ultramicrotome (Microm). Ultraviolet absorption spectra were measured from the sections after the resin in the section was removed with acetone (Yoshinaga et al., 1997). Ultraviolet absorption was surveyed in the range of 250 $400 \mathrm{~nm}$ in $2.5 \mathrm{~nm}$ steps for spot diameter $(0.5 \mu \mathrm{m})$ and a band width of an illuminating monochrometer $(5 \mathrm{~nm})$ using a microspectrophotometer (Carl Zeiss MPM-800). Three spectra were recorded from each cell type and 20-30 cells from the cambial zone to the pith were analyzed in vessel secondary walls (V-SW), fiber secondary walls (F-SW) and cell corner middle lamella located between fibers (FF-CC).

\section{Immunocytochemistry}

Antisera. Two specific polyclonal antibodies prepared and characterized as described in Joseleau and Ruel (1997) were used as antisera. They were directed, respectively, against: mixed guaiacyl/syringyl (GS) lignin polymer containing non-condensed interunit linkages $(\beta-0-4)$, and, mixed guaiacyl/syringyl lignin polymer containing condensed interunits.

Sample preparation for immunocytochemistry. Small slices (1 $\mathrm{mm}$ in thickness) obtained by freehand sectioning with a razor blade were fixed in a freshly prepared mixture of $0.1 \%$ glutaraldehyde $(\mathrm{v} / \mathrm{v}), 2 \%$ paraformaldehyde $(\mathrm{w} / \mathrm{v})$ in $0.05 \mathrm{M}$ phosphate buffer ( $\mathrm{pH}$ 7-7.2). After rinsing in phosphate buffer, 
they were dehydrated through a graded ethanol series up to $80 \%$ $(\mathrm{v} / \mathrm{v})$, then infiltrated and embedded in LR White resin (hard mixture, $T A A B)$ and polymerized for $24 \mathrm{~h}$ at $50^{\circ} \mathrm{C}$ as described earlier (Ruel et al., 1994).

Immunolabeling. In TEM, immunolabeling was done on ultrathin transverse sections ( $500 \AA$ ) floating downward in plastic rings passed on $50 \mu \mathrm{l}$ drops of reactives deposited on parafilm. The sections were first treated with $0.15 \mathrm{M}$ glycin in Tris- $\mathrm{HCl}$ buffer $0.01 \mathrm{M}, \mathrm{pH} 7.6$, containing $500 \mathrm{mM} \mathrm{NaCl}$, for blocking remaining aldehyde functions. This was followed by 2 min rinse (x5) on TBS500. Protein-protein interactions were blocked for 30 min by incubating on $5 \%(w / v)$ non-fat dried milk in TBS500. The sections were then incubated on each antiserum diluted 1: 50-1: 100 in the blocking buffer. Incubation time was $3 \mathrm{~h}$ at room temperature followed by one night at $4^{\circ} \mathrm{C}$. After four washes (2 min each) in TBS500 followed by three rinses in Tris- $\mathrm{HCl}$ buffer $(0.01 \mathrm{M}$ Tris- $\mathrm{HCl}, \mathrm{pH} 7.4-7.6)$, the sections were floated on the secondary marker [protein A-gold ( $\mathrm{pA} 5$ ) (Amersham)] diluted 1: 25 in Tris- $\mathrm{HCl}$ buffer containing $0.2 \%$ fish gelatin for $90 \mathrm{~min}$ at room temperature. They were washed five times ( $2 \mathrm{~min}$ each) in Tris- $\mathrm{HCl}$ buffer and three times in $\mathrm{H}_{2} \mathrm{O}$. The sections were then postfixed in $2.5 \%$ glutaraldehyde in $\mathrm{H}_{2} \mathrm{O}$ and washed three times in $\mathrm{H}_{2} \mathrm{O}$. At this stage, the diameter of the $5 \mathrm{~nm}$ gold particles was further enhanced using a silver enhancing kit from Amersham. Finally, thin sections were transferred on carbon-coated copper grids and poststained in $2.5 \%$ aqueous uranyl acetate. Observations were performed at $80 \mathrm{kV}$ with a Philips CM 200 Cryo-electron microscope.

All comparative immunolabeling experiments were carried out in parallel in order to keep the same experimental conditions (dilutions of antibodies, times of contact, etc.). Pre-immune serum for each antibody was assayed in the same conditions as described for the immunogold labeling.

\section{Cytochemical staining for polysaccharides in electron microscopy}

The polysaccharide moiety of the walls was contrasted on ultrathin sections by the periodic acid-thiocarbohydrazide-silver proteinate (PATAg) method modified for secondary walls by Ruel etal. (1981).

\section{Acknowledgements}

We wish to thank the European Community for its support (TIMBER "FAIR" programme (Contrat $n^{\circ}$ FAIR-CT95-0424)(DGXII) and COPOL 'Quality of life and Management of living resources' programme (Contrat $n^{\circ}$ QLSK-CT-2000-01493)), the CNRS, University Paul Sabatier and the Conseil regional Midi-Pyrénées for financial support and facilities. We also acknowledge $\mathrm{C}$. Guidice for typing a part of the manuscript.

\section{References}

Adler, E., Björkquist, K. and Häggroth, S. (1948) Uber die Ursache der Farbreaktionen des Holzes. Acta. Chem. Scand. 2, 93-94.

Chabannes, M., Barakate, A., Lapierre, C., Marita, J.M., Ralph, J., Pean, M., Danoun, S., Halpin, C., Grima-Pettenati, J., Boudet, A.M. (2001) Strong decrease in lignin content without significant alteration of plant development is induced by simultaneous down-regulation of cinnamoyl CoA., reductase
(CCR) and cinnamyl alcohol dehydrogenase (CAD) in tobacco plants. Plant $\mathrm{J}$.

Chapple, C., Vogt, T., Ellis, B. and Somerville, C. (1992) An Arabidopsis mutant defective in the general phenylpropanoid pathway. Plant Cell, 4, 1413-1424.

Chen, C., Meyermans, H., Burggraeve, B., De Rycke, R., Inoue, K., De Vleesschauwer, V., Steenackers, M., Van Montagu, M., Engler, G. and Boerjan, W. (2000) Cell-specific and conditional expression of Caffeoyl-Coenzyme A-3-O-Methyltransferase in Poplar. Plant Physiol. 123, 853-868.

Donaldson, L. (1994) Mechanical constraints on lignin deposition during lignification. Wood Sci. Technol. 28, 111-118.

Fergus, B.J. and Goring, D.A.I. (1970) The location of guaiacyl and syringyl lignins in birch xylem tissue. Holzforschung, 24, 113117.

Fukazawa, K. (1992) Ultraviolet microscopy. In: Methods in Lignin Chemistry (Lin, S. and Dence, C.W. eds). Springer-Verlag, pp. 110-121.

Gang, D.R., Costa, M.A., Fujita, M., Dinkova Kostova, A.T., Wang, H.B., Burlat, V., Martin, W., Sarkanen, S., Davin, L.B. and Lewis, N.G. (1999) Regiochemical control of monolignol radical coupling: a now paradigm for lignin and lignan biosynthesis. Chem. Biol. 6, 143-151.

Halpin, C., Knight, M., Foxon, G., Campbell, M., Boudet, A.M., Boon, J., Chabbert, B., Tollier, M. and Schuch, W. (1994) Manipulation of lignin quality by down-regulation of cinnamyl alcohol dehydrogenase. Plant J. 6, 339-350.

Houtman, C.J. and Atalla, R.H. (1995) Cellulose-lignin interactions - A computational study. Plant Physiol. 107, 977-984.

Jacquet, G., Pollet, B., Lapierre, C., Francesch, C., Rolando, C. and Faix, O. (1997) Thioacidolysis of enzymatic dehydrogenation polymers from $p$-hydroxyphenyl, guaiacyl and syringyl precursors. Holzforschung, 51, 349-354.

Joseleau, J.P. and Ruel, K. (1997) Study of lignification by noninvasive techniques in growing maize internodes - An investigation by Fourier transform infrared cross-polarization magic angle spinning C-13-nuclear magnetic resonance spectroscopy and immunocytochemical transmission electron microscopy. Plant Physiol. 114, 1123-1133.

Lev-Yadun, S. (2000) Cellular patterns in dicotyledonous woods: their regulation. In: Cell and Molecular Biology of Wood Formation (R. Savidge, J. Barnett and R. Napier, eds). Oxford, UK: Bios Scientific Publishers, pp. 315-324.

Musha, Y. and Goring, D. (1975) Distribution of syringyl and guaiacyl moieties in hardwoods as indicated by ultraviolet microscopy. Wood Sci. Technol. 9, 45-58.

Piquemal, J., Lapierre, C., Myton, K., Schuch, W., GrimaPettenati, J. and Boudet, A.M. (1998) Down-regulation of cinnamoyl-CoA reductase induces significant changes of lignin profiles in transgenic tobacco plants. Plant J. 13, 71-83.

Roussel, M.R. and Lim, C. (1995) Dynamic model of lignin growing in restricted spaces. Macromolecules, 28, 370-376.

Ruel, K., Faix, O. and Joseleau, J.P. (1994) New immunogold probes for studying the distribution of the different lignin types during plant cell wall biogenesis. J. Trace Microprobe Technical, 12, 247-265.

Ruel, K., Barnoud, F. and Eriksson, K.E. (1981) Micromorphological and ultrastructural aspects of spruce wood degradation by wild type Sporotrichum pulverulentum and its cellulase-less mutant Cel 44. Holzforschung, 35, 157-171.

Siegel, S. (1957) Non-enzymatic macromolecules as matrices in biological synthesis: the role of polysaccharides in peroxydasecatalysed lignin polymer from eugenol. J. Am. Chem. Soc. 79, 1628-1632. 
Terashima, N. and Fukushima, K. (1989) Biogenesis and structure of macromolecular lignin in the cell wall of tree xylem as studied by microautoradiography. In Plant Cell Wall Polymers, Biogenesis and Biodegradation (Lewis, N.G. and Paice, M.G., eds). Washington DC, UK: American Chemical Society Symposium Series 399, pp. 160-168.

Terashima, N., Fukushima, K., He, L., Takabe, K. (1993) Comprehensive model of the lignified plant cell wall. In Forage Cell Wall Structure and Digestibility (Jung, et al., ed.). Madison, WI, USA: ASA-CSSA-SSSA, pp. 247-269.

Terashima, N., Fukushima, K. and Takabe, K. (1986) Heterogeneity in formation of lignin. VIII. An autoradiographic study on the formation of guaiacyl and syringyl lignin in Magnolia kobus DC. Holzforschung, 40, 101-105.

\section{Abbreviations}

COMT: Caffeic acid/5-OH ferulic acid O-methyltransferase

CCoAMT: Caffeoyl coenzyme A 3-O methyltransferase

CCR: Cinnamoyl CoA reductase

CAD: Cinnamyl alcohol dehydrogenase

CCR.H: Transgenic tobacco line homozygous for the ccr antisense gene

CAD.H: Transgenic tobacco line homozygous for the cad antisense gene

Dt: Double transformant down-regulated for both CCR and CAD
Turner, S.R. and Somerville, C.R. (1997) Collapsed xylem phenotype of Arabidopsis identifies mutants deficient in cellulose deposition in the secondary cell wall. Plant Cell, 9, 689-701.

Yahiaoui, N., Marque, C., Myton, K.E., Negrel, J. and Boudet, A.M. (1998) Impact of different levels of cinnamyl alcohol dehydrogenase down-regulation on lignins of transgenic tobacco plants. Planta, 204, 8-15.

Yoshinaga, A., Fujita, M. and Saiki, H. (1997) Secondary wall thickening and lignification of oak xylem components during latewood formation. Mokuzai Gakkaishi, 43, 377-383.

Zhong, R., Taylor, J.J. and Y.e.Z.H. (1997) Disruption of interfascicular fiber differentiation in an Arabidopsis mutant. Plant Cell, 9, 2159-2170. 